\title{
Moulagen und Epithesen: Angewandte Kunst in der Medizin
} C. Löser $^{1}$
H. Ständer
S. Ständer

1. Arbeitstreffen der AGDV (Arbeitsgemeinschaft der Geschichte der Dermatologie und Venerologie) am 17.4. 2004, Münster

Moulages and Epithesis: Applied Art in Medicine - 1st Meeting of the AGDV
(Working Group for the History of Dermatology and Venerology)

Moulagen haben nach wie vor nicht nur eine Bedeutung als faszinierende, medizingeschichtliche Objekte, sondern auch ihren didaktischen Charakter erhalten. Bis heute stellt kein anderes Medium Hautkrankheiten so plastisch dar. Die jüngere Schwester der Moulage ist die Epithese. Damit werden entstellende Haut- und Gewebedefekte verdeckt, die häufig als Folge von Hauterkrankungen oder Traumen entstanden sind.

Die Arbeitsgemeinschaft für Geschichte der Dermatologie und Venerologie e.V. (AGDV) widmete sich in ihrer ersten Arbeitssitzung in Münster am 17. April 2004 diesem faszinierenden Themenkreis (Abb.1). Nach einer Begrüßung der zahlreichen Teilnehmer durch den Direktor der Münsteraner Hautklinik, Prof. Thomas Luger, erklärte der Vorsitzende der Arbeitsgemeinschaft, Prof. Albrecht Scholz aus Dresden, die Bedeutung des Themas für die Medizingeschichte und stellte mit Frau Elsbeth Stoiber aus Zürich den Ehrengast der Veranstaltung vor. Frau Stoiber ist die letzte Moulageurin, die 1953 auch in der Hautklinik Münster einige Moulagen angefertigt hatte. Da sie die Letzte ihrer Zunft ist, hat sie das streng gehütete Geheimrezept der Moulagenherstellung offenbart. Frau Stoiber berichtete als Zeitzeugin über ihr Leben und die bewegende Rolle, welche die Moulagen darin gespielt haben. Von ihrer Ausbildung, einer großen Studienreise nach Indien, und der Weiterentwicklung der Technik der Moulagenherstellung an bildhaften Beispielen war ebenso die Rede wie von nächtlichen Rettungsaktionen zur Entsorgung freigegebener Moulagen mit Hilfe von Freunden und deren Pferdegespannen. Schließlich schlug Frau Stoiber den Bogen zu den Epithesen, die sie anfangs aus Wachs und später aus Kunststoffen für Patienten in der ganzen Schweiz anfertigte. So folgten die Zuhörer gebannt

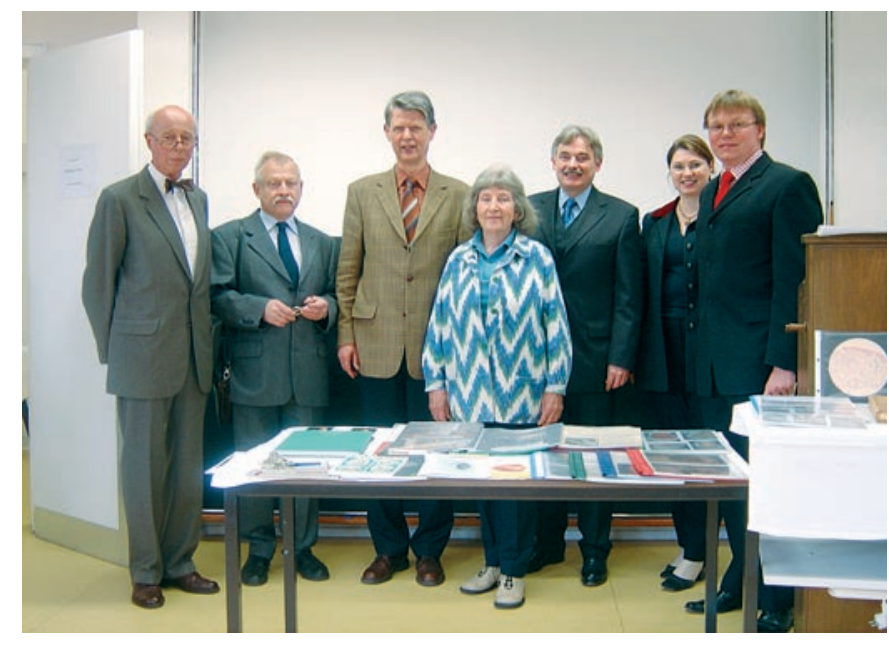

Abb. 1 Die Organisatoren und Redner der AGDV-Tagung am 17. 4. 2004. E. Macher, M. Hundeiker, A. Scholz, E. Stoiber, T. Luger, S. Ständer, H. Ständer (von links nach rechts).

ihrem ergreifenden und spannenden Vortrag über ihre 50-jährige Erfahrung. Prof. M. Hundeiker widmete sich dann in seinem fesselnden Referat der Frühzeit der Epithesen. Beginnend mit Nasen aus Gold für unglückliche Ritter, über Gelatineohren, welche sich die Patienten täglich neu gießen mussten, wurde ein umfassendes Bild der Entwicklung gezeichnet. Mit den heute üblichen modernen Kunststoffepithesen, die durch druckknopfartige Verbindungen am Knochen verankert werden, hat man, so Hundeiker, das Mittelalter der Epithesentechnik verlassen. Dabei 


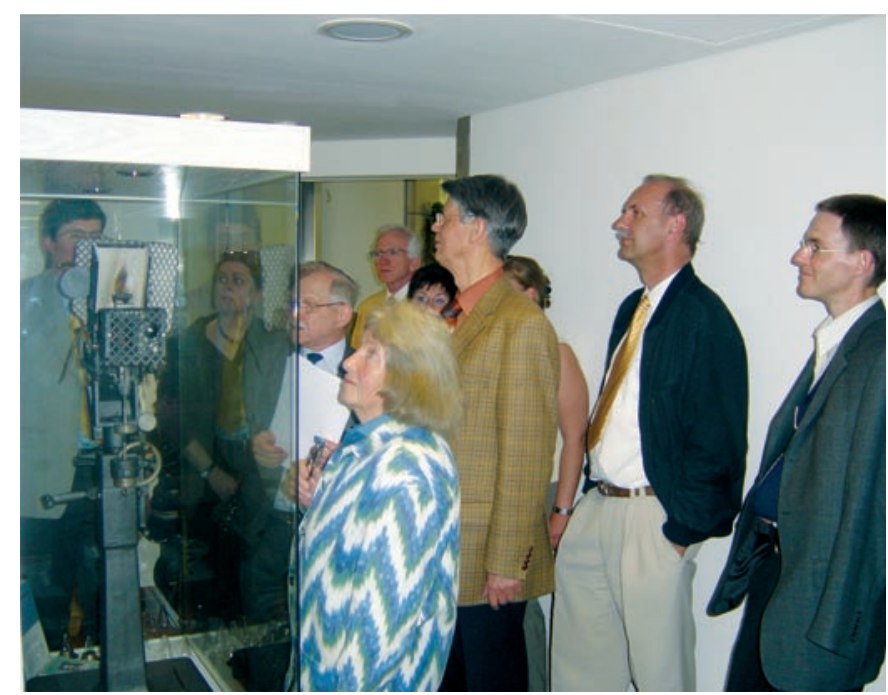

Abb. 2 Besichtigung der Hornheider Museumsvitrinen; im Bild links eine Finsen-Lampe zu erkennen.

erwähnte Hundeiker anekdotisch auch den für Münster offenbar noch heute nicht seltenen Verlust von Ohrmuscheln durch bissige Trunkenbolde. So konnte er den Zuhörern bei einem faszinierenden aber ernsten Thema auch heitere Seiten vermitteln. Ein weiterer Höhepunkt des Vormittags war ein kompetenter Überblick von Prof. A. Scholz über Dokumentationsformen in der Dermatologie, die vom Holzschnitt bis zum Video reichen.

Organisiert wurde die Veranstaltung von Dr. Hartmut Ständer und Dr. Sonja Ständer, die in ihrem Vortrag über den Aufbau und die Pflege der Münsteraner Moulagensammlung berichtete, welche heute 121 Präparate umfasst. An den Moulagen der Pocken oder des Milzbrandes lässt sich zeigen, dass diese Kunstwerke aus Wachs nicht an Relevanz verloren haben, wenn es beispielsweise darum geht, das Aussehen seltener Hauterkankungen auf weißer Haut zu vermitteln. Neben den informativen Vorträgen wurde die Veranstaltung durch eine Besichtigung der Münsteraner Moulagen sowie der historischen Instrumentesammlung der Fachklinik Hornheide unter der sachkundigen Führung durch Prof. Hundeiker (Abb. 2) abgerundet.

In ihrem didaktischen und medizinhistorischen Wert verkannt, wurden im letzten Jahrhundert durch fahrlässiges Desinteresse viele Moulagen zerstört. Engagierte Menschen haben an Orten wie Zürich, Dresden und Münster Sammlungen zusammengetragen, deren Besuch sich lohnt. Dort kann man sich am besten selbst davon überzeugen, welche Ästhetik dieses vernachlässigte Medium ausstrahlt.

\section{Buchbesprechung}

\section{Lehrbuch der Liposuktion}

G. Sattler, B. Sommer, C. W. Hanke

Stuttgart: Thieme, 2003. 274 S., 256 Abb., 17 Tab., Geb. 149,- $€$

ISBN 3-13-138141-8

Im letzten Jahrzehnt ist das Interesse an der Ästhetischen Dermatologie und damit auch an der Liposuktion sprunghaft gestiegen.

Mittlerweile hat sich die Liposuktion zu den am häufigsten durchgeführten ästhetisch-operativen Eingriffen entwickelt. Durch Verbesserung des Instrumentariums, der Tumeszenztechnik sowie den hohen Patientenzahlen konnte in den letzten Jahren ein hoher Sicherheitsstandard bei diesem kosmetischen Eingriff erreicht werden.

Das vorliegende Werk füllt eine Lücke im deutschsprachigen Raum aus. Die bisher verfügbaren Bücher zu diesem Thema sind in englischer Sprache verfasst. In einem leicht verständlichen Stil und klarer Gliederung präsentieren die Autoren den aktuellen Erfahrungs- und Wissensstand über die Liposuktion.

Neben der Darstellung der Anästhesieverfahren, Operationstechniken, der Indikationen, Kontraindikationen und Komplikationen der Liposuktion werden auch geschichtliche Aspekte sowie die Anatomie und Physiologie des Fettgewebes diskutiert.

Ein besonderer Lichtblick des Buches sind die vielen Farbaufnahmen, die die Lagerung des Patienten, die verwendeten Kanülen sowie das operative Vorgehen darstellen. Diese Abbildungen erleichtern auch dem Anfänger ein schnelleres und besseres Verständnis der Materie. Aber auch in der Liposuktion erfahrene Ärzte werden die vielen praxisnahen, sich auf eigene langjährige Erfahrung mit der Methode gründende Hinweise der Autoren dankbar zur Kenntnis nehmen.

Abgerundet wird das Buch durch Aspekte zu gutachterlichen Fragestellungen und Marketing sowie zu psychologischen Gesichtspunkten wie die Patientenzufriedenheit.

Zusammenfassend handelt es sich um ein Standardwerk der Liposuktion in deutscher Sprache, mitverfasst von G. Sattler, einem der Pioniere der Liposuktion in Deutschland. Das Buch ist für alle Anwender der Liposuktion sehr gut geeignet.

Verlag und Autoren sei eine weite Verbreitung der 1. Auflage gewünscht.

T. Karamfilov, Bad Säckingen 